\title{
Retained Mesoappendix Fat Nodule Mimicking Appendicitis
}

\author{
Sarma DR*, Thomas D and Watson M
}

Department of General Surgery, Daren't Valley Hospital, Dartford and Gravesham NHS Trust, UK

*Corresponding author: Sarma DR, M.B.B.S, D.N.B, M.R.C.S, Department of General Surgery, Daren’t Valley Hospital, Dartford and Gravesham NHS Trust, Darenth wood road, Dartford, DA2 8DA, UK, E-mail: dsarma@nhs.net

\author{
Citation: Sarma DR, Thomas D, Watson M (2016) Retained Mesoappendix Fat Nodule Mimicking \\ Appendicitis. J Gastroenterol Compl 1(1): 101
}

Received Date: July 10, 2015 Accepted Date: November 05, 2016 Published Date: November 09, 2016

\begin{abstract}
Appendicectomy is one of the commonest emergency surgery performed across the globe. Long term complication post appendicectomy are rare. Chronic pain post operatively can be a debilitating problem, often without identifiable underlying cause. We describe a rare instance where a patient suffering from ongoing pain post appendicectomy was noted to have ongoing chronic inflammation in a retained mesoappendicular fat nodule. With effective investigations and laparoscopic intervention, she was cured of her symptoms with excision of the mesoappendicular fat nodule.

Keywords: Post appendicectomy pain; Appendicolith; Retained fat necrosis; Appendicitis
\end{abstract}

\section{Introduction}

Diseases of the appendix can be acute, acute recurrent or chronic. Case reports in literature describe re-operation post appendisectomy at various time intervals for retained appendicolith. We present a rare case where re-operation two years post appendicectomy was performed for ongoing symptoms; and the cause noted to be a retained mesoappendicular fat nodule, mimicking as appendicitis again.

\section{Case report}

A fit and healthy 32 year old female underwent laparoscopic appendicectomy (standard technique, with 3 ports) for typical symptoms of acute appendicitis. Intra-operatively a turgid and inflamed appendix was noted, without any other intra abdominal pathology. The appendicular stump was ligated and the tip of the stump cauterised. Immediate post operative recovery was uneventful and she was symptom free and discharged within 24 hours of surgery. Histology of the non perforated appendix revealed significant acute inflammatory cell infiltrate and follicular hyperplasia.

Eight weeks from the operation, she presented back several times to the emergency department and outpatient clinic with ongoing right iliac fossa pain, anorexia and nausea. A plethora of investigations were advocated for her ongoing symptoms, including haematological tests for celiac disease, inflammatory bowel disease, repeated ultrasound of the abdomen, pelvis, and trans vaginal scans. Having found no cause of her ongoing symptoms in spite of being on analgesia and anti-inflammatory, she underwent a colonoscopy and multiple random biopsies were taken from her large bowel and terminal ileum. Needless to say, the colonoscopy was normal and there were no evidence of colitis, ileitis, dysplasia or malignancy in the biopsy specimen.

The patient having suffered with chronic symptoms for almost two years was consented for a diagnostic laparoscopy. She underwent axial imaging (Computer tomography scan) prior to re-laparoscopy, which was normal except for some fatty stranding at the ileo-caecal junction (Figure 1). Interestingly what was noted in the re-laparoscopy was a $2 x 3 \mathrm{~cm}$ mesoappendicular fat nodule at the ileo-caecal junction, adherent to the omentum, caecum and ileum (Figure 2 and 3). Rest of the intra-peritoneal structures were normal. This nodule was dissected free from the surrounding structures, excised and sent across for histopathological examinations. Remarkably patient had successful recovery and has been asymptomatic ever since, almost six months from her re-laparoscopic excision of the messoappendicular fat nodule. The histology revealed fibro fatty tissue with encapsulated fat necrosis, microcalcifications and scanty inflammatory cells. Retrospectively a diagnosis of retained mesoappendicular fat nodule necrosis was made to be the cause of her re presentation with symptoms mimicking acute appendicitis, two years post laparoscopic appendisectomy. 


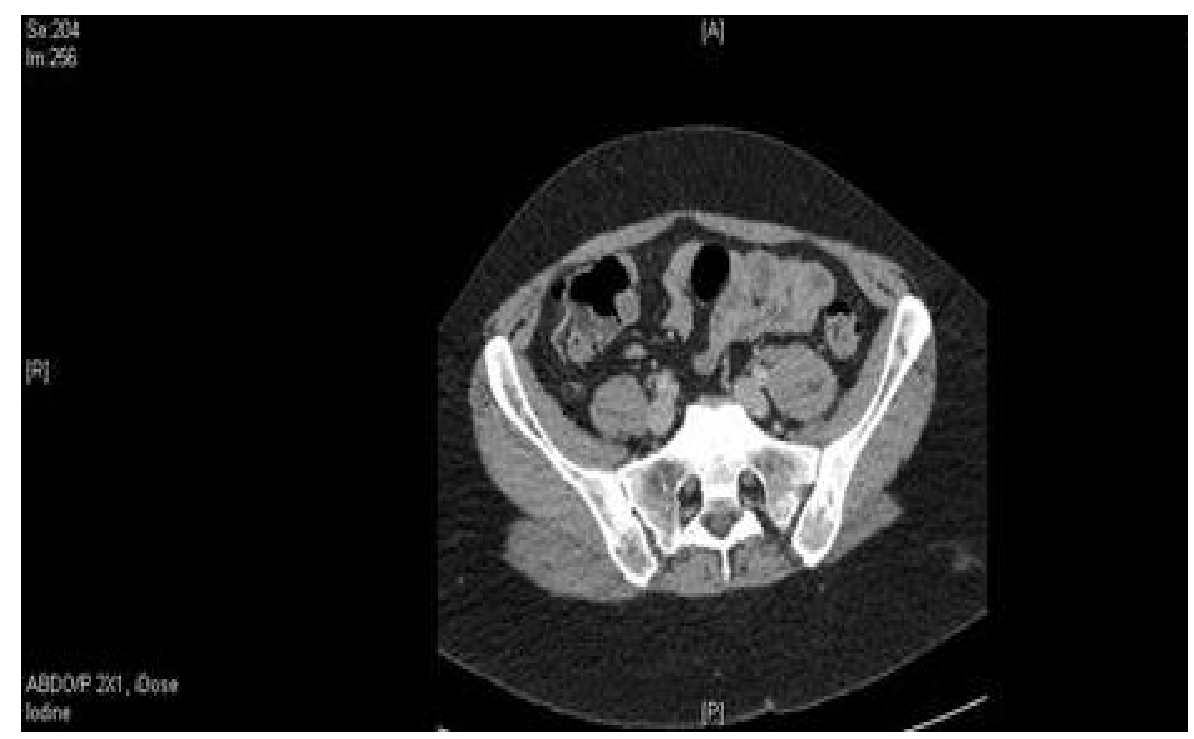

Figure 1: CT scan image showing fatty stranding at the ileo-caecal junction
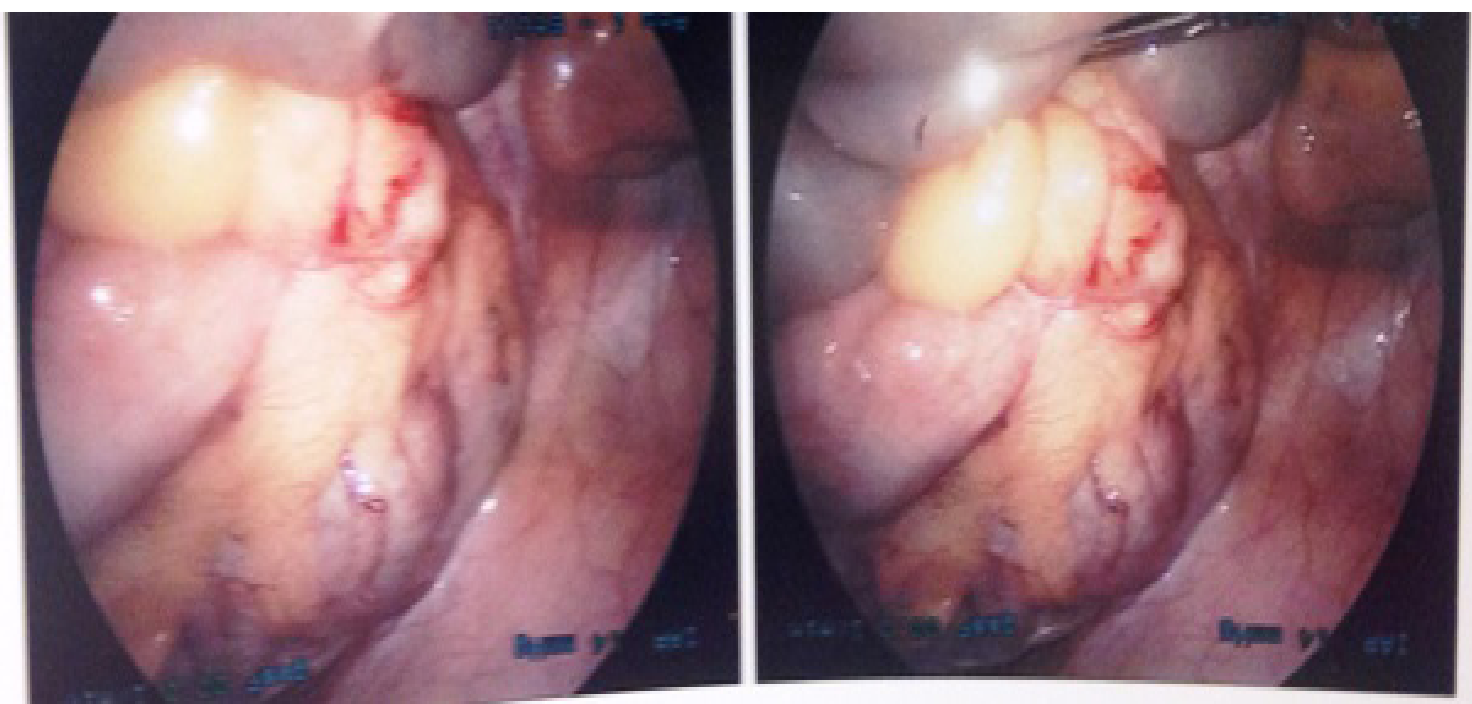

Figure 2: Intra operative finding in re-laparoscopy showing mesoappendicular fat nodule 


\section{Discussion}

There have been several anecdotal accounts of post appendisectomy pain in literature [1]. Case-series have reported several causes, such as retained appendicolith, missed diagnosis, stump appendicitis, ileal chron's disease and celiac diseases [2]. There is no report in literature about retained mesoappendicular fat nodule, with necrosis leading to such chronic symptoms. The advent of laparoscopy and its use as a diagnostic tool has revolutionized surgical practice. This report highlights laparoscopy as a diagnostic and therapeutic tool in the armamentarium of general surgeons. The mechanism of the chronic symptoms in this case report, is attributable to the ongoing chronic inflammatory process in the retained mesoappendicular fat, which was clearly distinct from any appendiceal tissue on microscopy. There are case studies in literature documenting, relief of abdominal symptoms even after removal of normal appendix, also on the other extreme there are reports of chronic abdominal pain post appendicectomy for an inflammed appendix. Even though it is difficult to pinpoint the exact mechanism involved in manifestation of the symptoms in our patient, there is enough evidence to suggest that the retained mesoappendicular nodule was the cause for it. What is reassuring from the patient follow up, is complete resolution of abdominal symptoms after the second laparoscopic intervention. The rarity of the diagnosis also throws light on the technical aspects of laparoscopic appendicectomy, ensuring there are no retained omentum/ fat/mesoappendicular devascularized tissues in the peritoneal cavity, which might lead to further intra abdominal problems, diagnostic dilemmas and re-operations.

\section{Conclusion}

Retained mesoappendicular fat necrosis as a cause of chronic pain post appendisectomy is rare, but an important feature to be considered in patients having ongoing symptoms mimicking appendicitis, post appendicectomy. The use of laparoscopy in such cases gives the added advantage of both diagnostic and therapeutic intervention.

\section{Acknowledgements}

There are no conflicts of interests. All the authors are in agreement with the content of the submission. There are no funding involved. Informed consent has been obtained from the patient for publication of the clinical images and information.

\section{References}

1. Hollerman JJ, Bernstein MA, Kottamasu SR, Sirr SA (1988) Acute recurrent appendicitis with appendicolith. Am J Emerg Med 6: 614-7.

2. Guillem P, Mulliez E, Proye C, Pattou F (2004) Retained appendicolith after laparoscopic appendectomy: the need for systematic double ligature of the appendiceal base. Surg Endosc 18: 717-8. 